\title{
A case report of disseminated nocardiosis with ocular involvement in a myasthenia gravis patient and literature review
}

\author{
Shuhui Wang ${ }^{1 *} \mathbb{D}$, Bin Jiang ${ }^{1}$, Yao Li ${ }^{1}$, Yanchang Shang ${ }^{2}$, Zhengshan Liu $^{3}$ and Yongbo Zhang ${ }^{1}$
}

\begin{abstract}
Background: Nocardiosis is a rare and life-threatening opportunistic infection in immunocompromised patients. Myasthenia gravis (MG) patients are potentially at risk of nocardia infection because of the use of immunosuppressive agents. To date, only 7 patients with MG have been reported to have nocardiosis. Disseminated nocardiosis with ocular involvement has not been reported in MG patients.

Case presentation: A 66-year-old man with MG who was receiving treatment with methylprednisolone and azathioprine was found to have a respiratory infection. He also had heterogeneous symptoms with skin, brain and ocular manifestations. Nocardia bacteria verified by the culture of puncture fluid, and a diagnosis of disseminated nocardiosis was made. Except for left eye blindness, the patient completely recovered from the disease with combination antibiotic therapy. To further understand nocardiosis in patients with MG, we reviewed the previous relevant literature. According to the literature, this is the first report of disseminated nocardiosis with ocular involvement in an MG patient.
\end{abstract}

Conclusions: MG patients with immunosuppressant treatments are potentially at risk of a rare nocardia infection, and a favourable prognosis can be achieved through early diagnosis and appropriate antibiotic therapy.

Keywords: Disseminated nocardiosis, Myasthenia gravis, Opportunistic infection, Case report

\section{Background}

Nocardiosis is an acute purulent or chronic granulomatous disease caused by infection of nocardia bacteria, a gram-positive branching rod-shaped aerobic bacterium from the genus Actinomyces [1, 2]. Nocardia is an opportunistic pathogen with a relatively low incidence, and it is usually infectious in immunocompromised patients, such as those with autoimmune disease or those receiving immunosuppressive treatments. They can cause local or disseminated nocardiosis in humans. Patients with myasthenia gravis (MG) are commonly treated with immunosuppressive agents for a long time and are potentially at risk of nocardia infection.

Nocardiosis is a life-threatening infectious disease, especially in patients who cannot be treated appropriately in the early stage of the disease. However, the heterogeneous

\footnotetext{
*Correspondence: drwangshuhui@163.com

'Department of Neurology, Beijing Friendship Hospital, Capital Medical

University, Beijing 100050, China

Full list of author information is available at the end of the article
}

clinical presentations of affected patients and laboratory test limitations make the diagnosis of nocardiosis very difficult. Therefore, it is important for physicians to diagnose the disease and treat the patients in a timely manner. Here, we present a case of disseminated nocardiosis in an MG patient in whom multiple systems were involved. Based on our findings, we also conducted a literature review.

\section{Case presentation}

A 66-year-old Chinese man presented with fever, cough, dyspnoea and lumbodynia after falling from exercise equipment on July 6, 2015. He had a medical history of blood hypertension and diabetes mellitus. In 2014, he was hospitalized because of fluctuating ptosis and dysphagia. The fatigue test and neostigmine test were positive. Slow frequency repetitive nerve stimulation $(3 \mathrm{~Hz})$ on the bilateral facial nerves showed that the compound muscle action potential (CAMP) decrement was more than $15 \%$. An immunological serum test showed that the 
patient was negative for the acetylcholine receptor (AChR) and muscle-specific kinase (MusK) antibodies but positive for Titin and ryanodine receptor (RyR) antibodies. Thoracic enhanced computed tomography (CT) did not find an abnormal thymus. The patient was diagnosed with generalized MG and received glucocorticoids and pyridostigmine therapy. The maximum dose of methylprednisolone was $56 \mathrm{mg}$ per day, which was subsequently tapered to $20 \mathrm{mg}$ per day. In addition, he took $100 \mathrm{mg}$ azathioprine daily for MG therapy.

A physical examination revealed that the patient had a temperature of 38 degrees centigrade. Other vital signs were in the normal range. There were multiple rales in the bilateral lungs on auscultation. Multiple, irregular, and tender masses were found on the patient's chest, back, neck, and right limbs. These masses had a high temperature and were red in colour. There was an infectious ulcer mass on the right lower limb. Except for weakness in the right lower limb (5-/5), there were no other abnormal findings on a neurological examination.

Routine laboratory investigations, including routine blood tests, hepatic and renal functions, electrolyte and coagulation function, were in the normal range. Antinuclear antibody (ANA), extractable nuclear antibody (ENA), and neoplastic marker tests were in the normal range.
Immunoglobulin (Ig) and complement (C) levels were checked twice. The patient's levels of $\mathrm{IgG} / \mathrm{IgA} / \mathrm{C} 3 / \mathrm{C} 4$ were normal, but his levels of IgM were $25 \mathrm{mg} / \mathrm{dL}$ and $25.7 \mathrm{mg} /$ dL (normal limit, 40-230 mg/dL). The proportions of lymphocyte subgroups (CD3, CD4, CD8, CD4/CD8, CD19, and CD16 + CD56) were normal. Respiratory virus screening was negative. His level of $C$ reactive protein (CRP) was $37 \mathrm{mg} / \mathrm{L}$ (normal range $<5 \mathrm{mg} / \mathrm{L}$ ). Tuberculosis infection $\mathrm{T}$ lymphocytes and anti-tuberculosis antibody were not positive. Antibodies against hepatitis B virus (HBV), hepatitis C virus (HCV), human immunodeficiency virus (HIV) and syphilis were negative.

A lumbar spine X-ray scan showed compression fractures in the L1 and L2 vertebral bodies. A neck CT scan found a mass on the left, and a diagnosis of swollen lymph node, abscess or tumour was considered (Fig. 1a). A chest CT scan revealed multiple nodular and patchy shadows in the bilateral lungs and pleural as well as pericardial effusion (Fig. 1b-c). An abdominal CT scan revealed gallstones and a small cyst on the right kidney.

The patient was diagnosed with pneumonia and received moxifloxacin treatment. The patient's body temperature gradually returned to the normal range, and cough and dyspnoea were also relieved after treatment. However, he had a fever with chill, heavy dyspnoea, headache, and pain in
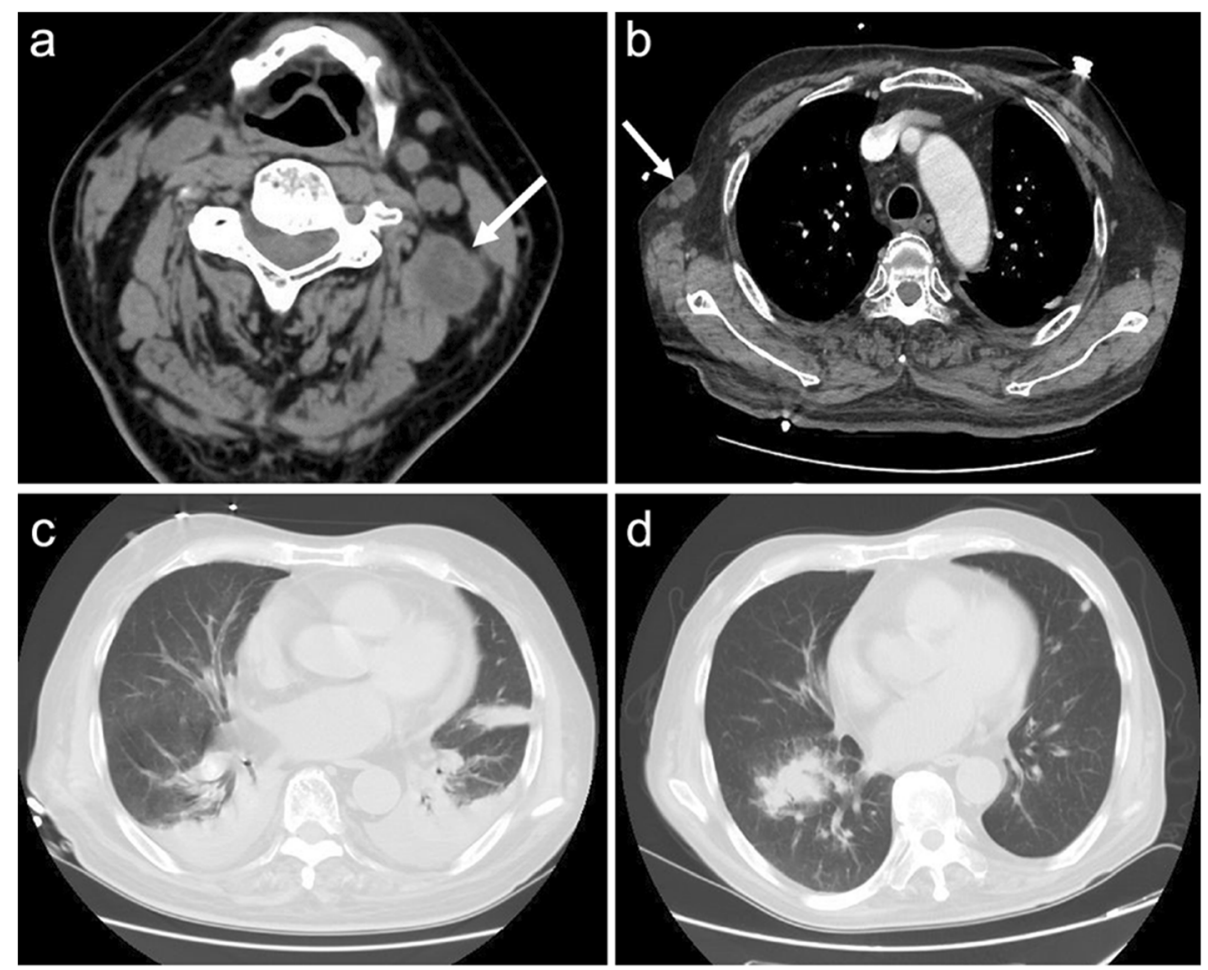

Fig. $1 C T$ images of the neck and chest on the day of admission to hospital $(\mathbf{a}, \mathbf{b}, \mathbf{c})$ : a CT scan showing a mass on the left of the neck, arrow shown in (a). CT of the chest showing the subcutaneous nodes, with the arrow shown in (b). There were multiple nodular or patchy shadows in the bilateral lungs and pleural and pericardial effusion (c). One month after treatment with SMZ-TMP, a repeated chest CT (d) showed pleural effusion and that the lesions in the left lung had disappeared while the lesions in the right lung had become more apparent 
the right leg after 20 days of treatment. He also felt great pain in the left eye, and his vision became blurred and rapidly aggravated to blindness. For differential diagnoses, both brain CT scan and magnetic resonance imaging (MRI) were performed. The CT scan showed multiple low-intensity lesions in different brain areas, and MRI revealed marked multiple abnormalities in the bilateral cerebrum and cerebellum with low signal intensity on T1-weighted images as well as high signal intensity on T2-weighted images. After administration of gadolinium contrast material, these lesions demonstrated ring enhancement (Fig. 2a-d). Accordingly, a diagnosis of abscess or multiple brain metastases was considered. Importantly, MRI of the orbit showed an abnormal enhanced lesion behind the left eyeball and retinal detachment (Fig. 3a-c). To identify the characteristics of these lesions, we performed percutaneous drainage from the mass on the left neck guided by ultrasound. Nocardia bacteria were found in a culture of puncture fluid, confirming the diagnosis of disseminated nocardiosis.

After the diagnosis of nocardiosis, the patient was treated with trimethoprim-sulfamethoxazole (TMP-SMX) tablets (TMP0.08 g + SMX0.4 g/per tablet, 2 pills every $8 \mathrm{~h}$ ). Moreover, imipenem/meropenem/ceftriaxone and sporanox were also given for the bacteria and fungus infection. Two weeks later, the patient was found to have leukocytopenia and underwent a bone marrow puncture examination. The results indicated a suspected diagnosis of myelodysplastic syndrome (MDS). Therapy with TMP-SMX tablets was adjusted to 1 pill every $12 \mathrm{~h}$ plus injection with etimicin sulfate. Azathioprine therapy was replaced with berbamine to treat the leukocytopenia. Three weeks later, the patient was treated with TMPSMX tablets alone (1 pill every $12 \mathrm{~h}$ ). After this treatment, except for the left eye blindness, all of his symptoms, including fever, dyspnoea, headache, and pain in the leg and left eye, gradually remitted. In addition, 1 month of TMPSMX treatment also reduced pleural effusion and bilateral lung lesions based on repeat chest CT results (Fig. 1d). After one and a half months of TMP-SMX treatment, multiple brain lesions had decreased or even disappeared based on a repeat brain MRI (Fig. 2 e-h). The patient was continuously treated on an out-patient basis with TMPSMX tablets ( 1 pill every $12 \mathrm{~h}$ ) for one and a half years. His blood cells were monitored every 2 weeks. Six months after discharge, the subcutaneous masses had disappeared, and a repeat brain MRI showed that the encephalic lesions had also disappeared. At that point, the dosage of methylprednisolone was tapered slowly until it was stopped because of the improvement observed in his MG, and there were no recurrent syndromes at the 2-year follow-up.

\section{Discussion and conclusions}

In general, nocardia bacteria are opportunistic bacteria that mainly infect immunocompromised patients. Although MG is an autoimmune disease that requires immunosuppressive treatment, nocardia infection is rarely observed in these patients. We performed the literature review by searching the MEDLINE database using the
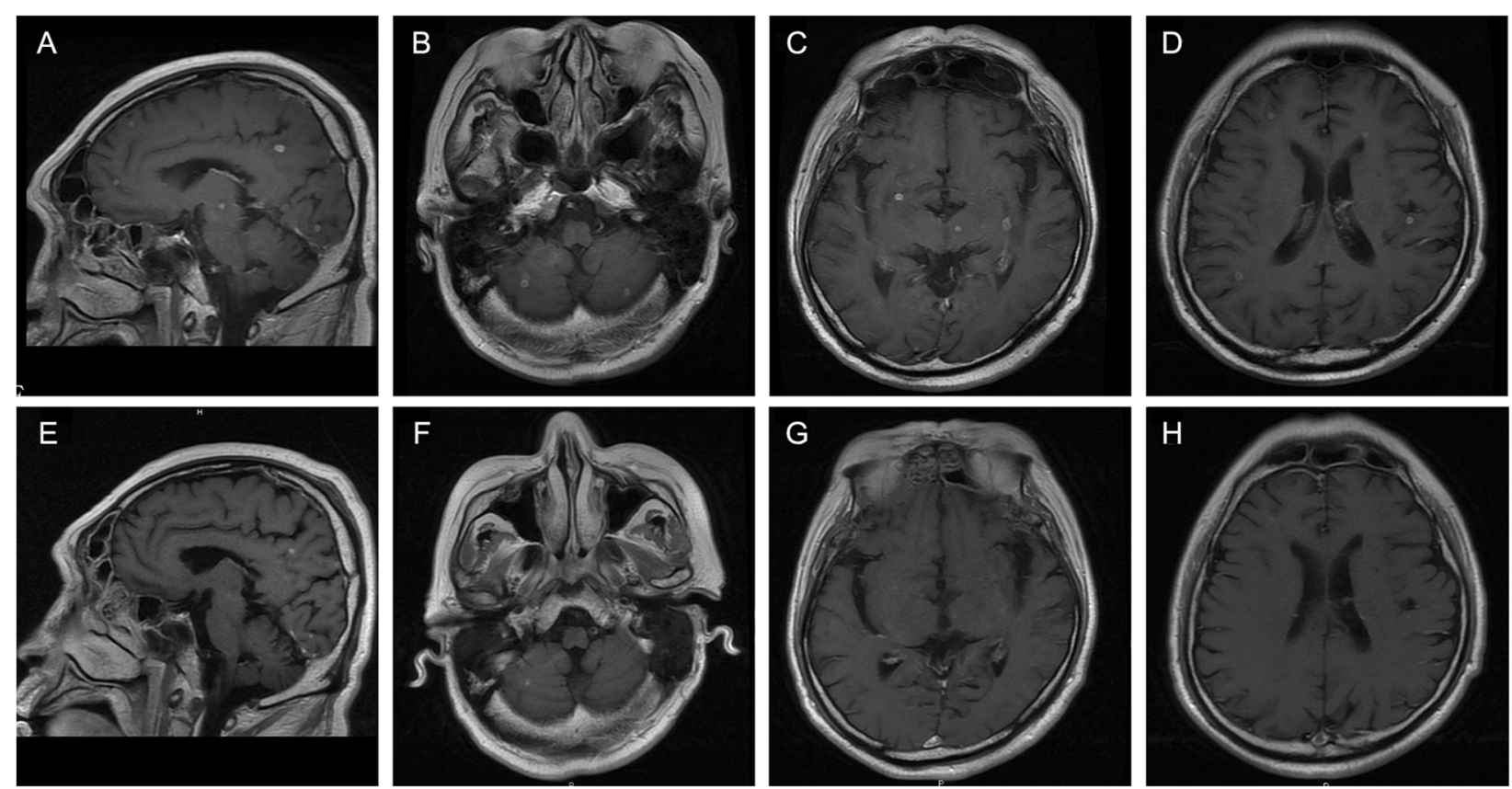

Fig. 2 Magnetic resonance imaging of the brain. Gadolinium contrast T1 images, with sagittal reformat (a) and axial reformat (b, c, d) images of the brain showing ring-enhancing lesions in the bilateral brain and cerebellum. $\mathbf{e}-\mathbf{h}$ : Reviewed MRI scan of the brain showing that some lesions had decreased and some had disappeared after one and half months of SMZ treatment 

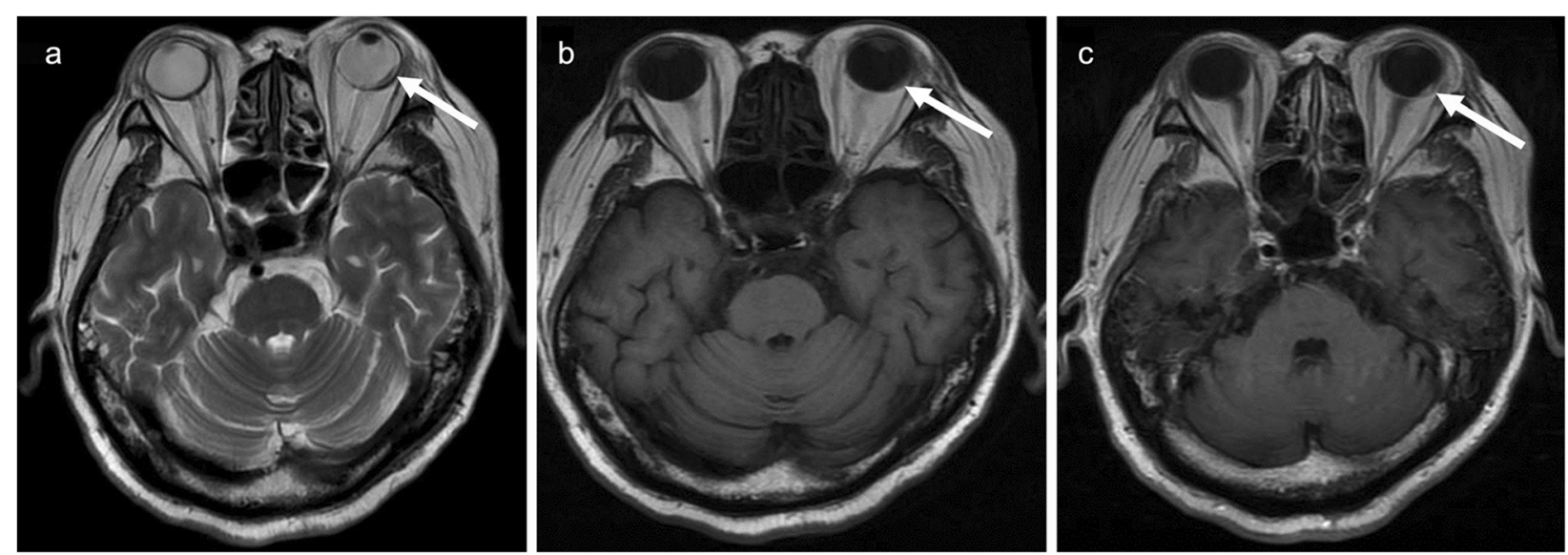

Fig. 3 Magnetic resonance imaging of the orbit. An abnormal lesion was noted behind the left eye (shown on arrow) with a high-intensity signal on T2-weighted images (a), low signal intensity on T1-weighted images (b) and high signal intensity on gadolinium contrast T1-weighted images (c)

key words "nocardia," "nocardiosis, "and "myasthenia gravis." We found that up until our search, only 7 case reports of MG patients with nocardiosis had been reported [3-9]. To improve understanding of the disease process, we summarized the clinical characteristics of these patients, and these are listed in Table 1. Seven out of 8 patients had a good prognosis. All patients were treated with glucocorticoids and other immunosuppressants. Three patients had thymoma, and 1 patient had Good's syndrome. There was no clear relationship between nocardia infection and MG or thymoma. However, patients with Good's syndrome, who usually have thymoma and immunodeficiency, easily acquire opportunistic infections. Although our patient had low IgM levels, he had no thymoma and normal levels of lymphocyte subgroups, and a diagnosis of Good's syndrome was therefore not considered. Repeated immunoglobulin tests showed that he had low levels of IgM and normal levels of IgG, IgA and lymphocyte subgroups. Selective immunoglobulin $\mathrm{M}$ deficiency (sIgMD) was considered in our patient and is a rare primary immunodeficiency disease characterized by low levels of IgM (below two standard deviations of the mean) in association with infection and normal levels of IgG and IgA [10]. In addition, our patient had the history of diabetes for many years. Therefore, immunosuppressant use, sIgMD and diabetes were the predisposing factors for nocardia infection in our patient. We concluded the data in Table 1. The following factors may be preconditions for nocardia infection in MG patients. The primary factor was the use of immunomodulator and/or immunosuppressive agents, consistent with numerous previous studies [11, 12]. The second factor was age. We found that MG patients with nocardia infection were 49-79 years old, indicating that older patients with MG might be more susceptible to nocardiosis. This is consistent with the fact that older MG patients have a higher incidence of tumours, such as thymoma, and low immunity. The last factor is gender, as supported by our finding that 7 out of 8 MG patients with nocardia infection were male patients. In summary, older male MG patients being treated with immunomodulator and/or immunosuppressive agents might represent a population that is susceptible to nocardia infection.

The clinical manifestations of nocardia infections are very heterogeneous and nonspecific. Lung, brain and skin are the most commonly affected sites [13]. All 8 patients had lung lesions. The infection also involved the muscles, heart, and kidneys. In addition to lung, brain and skin lesions, our patient also had ocular lesions. To the best of our knowledge, this is the first case report of an MG patient with disseminated nocardiosis with ocular lesions.

Ocular tissue is an unusual site for disseminated nocardiosis, and ocular infection is generally diagnosed as local nocardiosis, with presents a keratitis or endophthalmitis resulting from ocular trauma or surgery [14]. Occasionally, ocular infection might also be caused by haematogenous dissemination via the choroidal circulation [15]. The ocular nocardia infection that occurred in our patient may have been caused by haematogenous spread because the patient did not have eye trauma or a history of surgery and he had no abnormal signs in his eyeball. The prognosis of ocular nocardiosis is generally poor. Blindness is a common consequence, and ophthalmectomy is performed in approximately $30 \%$ of these patients. For these reasons, regular ophthalmologic screening should be performed in patients with suspected disseminated nocardiosis $[15,16]$. In our patient, the ocular lesion was located behind the left eyeball, 


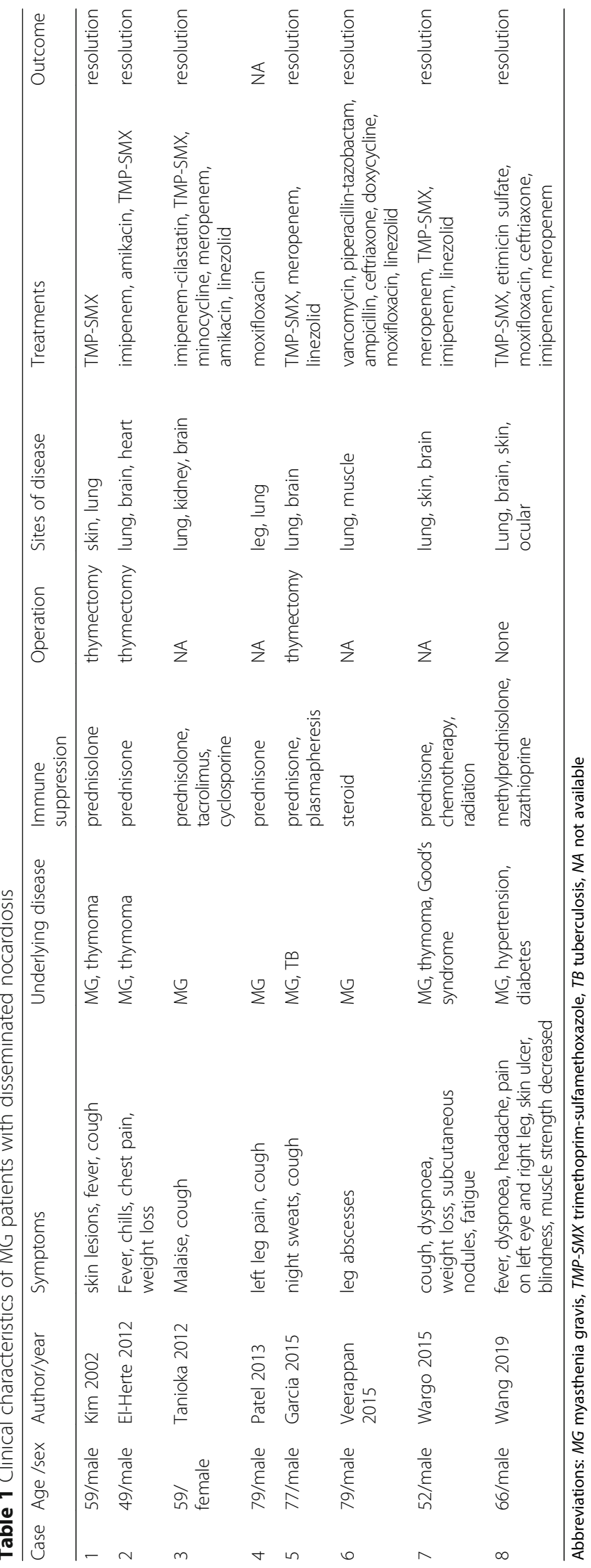


which finally led to retinal detachment. After he received suitable treatment, the ocular lesion completely vanished, but his vision was not restored.

Due to the paucity of trials, there are no formal guidelines to direct drug choice and treatment duration in nocardiosis. Most clinicians agree that CNS nocardiosis warrants a long course of treatment, with 12 months commonly recommended [17]. Empirical treatment of disseminated nocardiosis usually involves three antibiotics, including imipenem or ceftriaxone, TMP-SMX, and amikacin. TMP-SMX is thought to be the cornerstone of treatment for nocardia infections and is also the drug of choice for cerebral nocardiosis due to its good penetration into the CNS. Other drugs, including meropenem, cefotaxime, minocycline, moxifloxacin, levofloxacin, linezolid, tigecycline, and amoxicillin/clavulanic acid, are also used for the treatment of these patients [12]. MG patients should be treated with the proper antibiotics because some antibiotics can aggravate the disease. Patients with nocardiosis often have an underlying autoimmune disease or are receiving immunosuppressive treatment. Therefore, a combination of antibiotics is recommended in the beginning, and a single drug can be maintained after the clinical symptoms are relieved [12]. Immunosuppressive therapy will increase the risk of infection and the difficulty of treating infection in patients with MG. The use of immunosuppressants in MG patients with infections is an important issue. By reviewing the literature [18] and combining our findings with our own clinical practice experience, we cautiously suggest that if the infection can be controlled, immunosuppressive therapy can be continued in MG patients. However, when an infection is hard to control with administration of the proper antibiotics and becomes life-threatening, physicians should reduce the dose of immunosuppressants or even stop it. We stopped the use of azathioprine and continued a tapered dose of methylprednisolone in our patient when he developed leukocytopenia.

Because of the nonspecific manifestations of nocardiosis, most patients with nocardia infection are not diagnosed in the early stage of the disease, and it normally takes from 42 days to 12 months after the appearance of symptoms to achieve a clear diagnosis $[19,20]$; this causes a substantial physical and emotional burden in these patients. The prognosis of nocardiosis depends on the location and severity of the infection as well as the overall condition of the patient. The curable rate of pulmonary nocardiosis is approximately $90 \%$ with timely treatment, while the one-year mortality rate is high in patients with CNS involvement. The mortality rate is 10 -fold higher in patients with solid organ transplantation from nocardiosis than in those without $[11,12]$. None of the eight patients included in Table 1 had a history of organ transplantation. Although 4 of the 8 patients had CNS lesions, these lesions were relatively small, the symptoms were mild, and the diagnosis and treatment were timely, and this may have been the reasons for their good prognosis.

In conclusion, nocardiosis is a life-threatening infectious disease, and diagnosis in the early stage and appropriate antibiotic therapy are crucial to prognosis. Ocular involvement is rare in disseminated nocardiosis, and patients who are suspected to have disseminated nocardiosis should receive ophthalmologic screening. Neurological physicians should be aware of nocardia infection in MG patients.

\section{Abbreviations \\ AChR: Acetylcholine receptor; ANA: Antinuclear antibody; C: Complement; CAMP: Compound muscle action potential; CNS: Central nervous system; CRP: C reactive protein; CT: Computed tomography; ENA: Extractable nuclear antibody; HBV: Hepatitis B virus; HCV: Hepatitis C virus; HIV: Human immunodeficiency virus; Ig: Immunoglobulin; MDS: Myelodysplastic syndrome; MG: Myasthenia gravis; MRI: Magnetic resonance imaging; MusK: Muscle-specific kinase; NA: Not available; RyR: Ryanodine receptor; slgMD: selective immunoglobulin M deficiency; TB: Tuberculosis; TMP- SMX: Trimethoprim-sulfamethoxazole}

\section{Acknowledgements}

We thank Prof. Henry Kaminski (The George Washington University) for his valuable suggestions regarding the writing of the paper.

\section{Authors' contributions}

SHW: patient examination, medical data collection, and article preparation; BJ and YL: patient examination and medical data collection; YCS and ZSL: medical data analysis, article preparation, and correction and translation; YBZ: medical and scientific consultation and article preparation. All authors read and approved the final manuscript.

\section{Funding}

This work was supported by the Research Foundation of Beijing Friendship Hospital, Capital Medical University (No. yyqdkt2018-30).

\section{Availability of data and materials}

The datasets generated during and/or analysed during the current study are available from the corresponding author on reasonable request.

Ethics approval and consent to participate

The institutional review board of Beijing Friendship Hospital approved this case report. Written informed consent was obtained from the patient for the publication of the present case report.

\section{Consent for publication}

Written informed consent was obtained from the patient for publication of this case report and any accompanying images. A copy of the written consent is available for review by the Editor-in-Chief of this journal.

\section{Competing interests}

The authors declare that the research was conducted in the absence of any commercial or financial relationships that could be construed as a potential conflict of interest.

\section{Author details}

${ }^{1}$ Department of Neurology, Beijing Friendship Hospital, Capital Medical University, Beijing 100050, China. ${ }^{2}$ Department of Geriatric Neurology, Chinese People's Liberation Army General Hospital, National Clinical Research Center for Geriatric Diseases, Beijing 100853, China. ${ }^{3}$ Department of Neurology and Center for Translational Neuromedicine, University of Rochester Medical Center, New York, NY, USA. 
Received: 26 March 2019 Accepted: 9 October 2019

Published online: 21 October 2019

\section{References}

1. McNeil MM, Brown JM. The medically important aerobic actinomycetes: epidemiology and microbiology. Clin Microbiol Rev. 1994;7(3):357-417. https://doi.org/10.1128/cmr.7.3.357.

2. Takayanagi N, Kagiyama N, Ishiguro T, Tokunaga D, Sugita Y. Etiology and outcome of community-acquired lung abscess. Respiration. 2010;80(2):98105. https://doi.org/10.1159/000312404.

3. Kim CY, Kim TH, Lee WS, Lee AY. Reactive cutaneous cytophagocytosis in nocardiosis. J Korean Med Sci. 2002;17(2):279-82. https://doi.org/10.3346/ jkms.2002.17.2.279.

4. El-Herte RI, Kanj SS, Araj GF, Chami H, Gharzuddine W. First report of Nocardia asiatica presenting as an anterior Mediastinal mass in a patient with myasthenia gravis: a case report and review of the literature. Case Rep Infect Dis. 2012;2012:325767. https://doi.org/10.1155/2012/325767.

5. Tanioka K, Nagao M, Yamamoto M, Matsumura Y, Tabu H, Matsushima A, et al. Disseminated Nocardia farcinica infection in a patient with myasthenia gravis successfully treated by linezolid: a case report and literature review. J Infect Chemother. 2012;18(3):390-4. https://doi.org/10.1007/s10156-011-0315-1.

6. Patel N, Baker SM, Fernandes Filho JA. Leg pain in a patient with myasthenia gravis. J Gen Intern Med. 2013;28 11:1523; doi: https://doi.org/ 10.1007/s11606-013-2445-X.

7. Garcia RR, Bhanot N, Min Z. A mimic's imitator: a cavitary pneumonia in a myasthenic patient with history of tuberculosis. BMJ Case Rep. 2015;2015. https://doi.org/10.1136/bcr-2015-210264.

8. Veerappan Kandasamy V, Nagabandi A, Horowitz EA, Vivekanandan R. Multidrug-resistant Nocardia pseudobrasiliensis presenting as multiple muscle abscesses. BMJ Case Rep. 2015;2015. https://doi.org/10.1136/bcr-2014-205262.

9. Wargo JJ, Kim AH, Hart A, Berg A. It took a village: Good's syndrome. Am J Med. 2015;128(7):699-701. https://doi.org/10.1016/j.amjmed.2015.03.002.

10. Gupta S, Gupta A. Selective IgM deficiency-an underestimated primary immunodeficiency. Front Immunol. 2017;8:1056. https://doi.org/10.3389/ fimmu.2017.01056.

11. Lebeaux D, Freund R, van Delden C, Guillot H, Marbus SD, Matignon M, et al. Outcome and treatment of Nocardiosis after solid organ transplantation: new insights from a European study. Clin Infect Dis. 2017; 64(10):1396-405. https://doi.org/10.1093/cid/cix124.

12. Wilson JW. Nocardiosis: updates and clinical overview. Mayo Clin Proc. 2012; 87(4):403-7. https://doi.org/10.1016/j.mayocp.2011.11.016.

13. Beaman BL, Beaman L. Nocardia species: host-parasite relationships. Clin Microbiol Rev. 1994;7(2):213-64. https://doi.org/10.1128/cmr.7.2.213.

14. Sharma D, Mathur U, Gour A, Acharya M, Gupta N, Sapra N. Nocardia infection following intraocular surgery: report of seven cases from a tertiary eye hospital. Indian J Ophthalmol. 2017;65(5):371-5. https://doi.org/10.4103/ ijo. IJO_564_16.

15. Nishizawa A, Hirose M, Nagata Y, Takeuchi M, Satoh T. Disseminated cutaneous nocardiosis with ocular involvement. J Eur Acad Dermatol Venereol. 2017;31(11):e488-e9. https://doi.org/10.1111/jdv.14328.

16. Eisenberg MA, Wilker SC. Nocardia asteroides subretinal abscess in patient with acute myelogenous leukemia after allogeneic stem cell transplant. Retin Cases Brief Rep. 2014;8(2):113-5. https://doi.org/10.1097/ICB. 0000000000000017.

17. Ambrosioni J, Lew D, Garbino J. Nocardiosis: updated clinical review and experience at a tertiary center. Infection. 2010;38(2):89-97. https://doi.org/10. 1007/s15010-009-9193-9.

18. Gilhus NE, Romi F, Hong Y, Skeie GO. Myasthenia gravis and infectious disease. J Neurol. 2018;265(6):1251-8. https://doi.org/10.1007/s00415-018-8751-9.

19. Martinez R, Reyes S, Menendez R. Pulmonary nocardiosis: risk factors, clinical features, diagnosis and prognosis. Curr Opin Pulm Med. 2008;14(3):219-27. https://doi.org/10.1097/MCP.0b013e3282f85dd3.

20. Rafiei N, Peri AM, Righi E, Harris P, Paterson DL. Central nervous system nocardiosis in Queensland: A report of 20 cases and review of the literature. Medicine (Baltimore). 2016;95, e5255(46). https://doi.org/10.1097/MD. 0000000000005255.

\section{Publisher's Note}

Springer Nature remains neutral with regard to jurisdictional claims in published maps and institutional affiliations.

Ready to submit your research? Choose BMC and benefit from:

- fast, convenient online submission

- thorough peer review by experienced researchers in your field

- rapid publication on acceptance

- support for research data, including large and complex data types

- gold Open Access which fosters wider collaboration and increased citations

- maximum visibility for your research: over $100 \mathrm{M}$ website views per year

At BMC, research is always in progress.

Learn more biomedcentral.com/submissions 